\title{
Two-Dimensional Mn-Co LDH/Graphene Composite towards High-Performance Water Splitting
}

\author{
Jian Bao ${ }^{1}{ }^{\mathbb{D}}$, Junfeng Xie ${ }^{2, *} \mathbb{1}$, Fengcai Lei ${ }^{2}$, Zhaolong Wang ${ }^{1}$, Wenjun Liu ${ }^{1}, \mathrm{Li} \mathrm{Xu}^{1}$, \\ Meili Guan ${ }^{1}$, Yan Zhao ${ }^{1}$ and Huaming $\mathrm{Li}^{1}{ }^{1, *}$ \\ 1 Institute for Energy Research, School of Chemistry and Chemical Engineering, Jiangsu University, \\ Zhenjiang 212013, China; baojian@ujs.edu.cn (J.B.); ZhaolongW@hotmail.com (Z.W.); \\ Liuwenjun_ujs@163.com (W.L.); xulichem@ujs.edu.cn (L.X.); mlguan@ujs.edu.cn (M.G.); \\ yanzhao@ujs.edu.cn (Y.Z.) \\ 2 College of Chemistry, Chemical Engineering and Materials Science, Key Laboratory of Molecular and Nano \\ Probes (Ministry of Education), Collaborative Innovation Center of Functionalized Probes for Chemical \\ Imaging in Universities of Shandong, Institute of Molecular and Nano Science, \\ Shandong Normal University, Jinan 250014, China; leifc@sdnu.edu.cn \\ * Correspondence: xiejf@sdnu.edu.cn (J.X.); lhm@ujs.edu.cn (H.L.); \\ Tel.: +86-0531-8618-2538 (J.X.); +86-0511-8879-9500 (H.L.)
}

Received: 24 July 2018; Accepted: 23 August 2018; Published: 28 August 2018

\begin{abstract}
The oxygen evolution reaction (OER) is a complex multi-step four-electron process showing sluggish kinetics. Layered double hydroxides (LDH) were reported as promising catalysts for the OER, but their low electrical conductivity restricts their widespread applications. To overcome this problem, a composite material containing Mn-Co LDH ultrathin nanosheet and highly conductive graphene was synthesized for the first time. Benefited from the high electrocatalytic activity and the superior charge transfer ability induced by these components, the new material shows superior OER activity. Used as the OER catalyst, a high current density of $461 \mathrm{~mA} \mathrm{~cm}{ }^{-2}$ at $2.0 \mathrm{~V}$ vs. RHE (reversible hydrogen electrode) was measured besides shows a low overpotential of $0.33 \mathrm{~V}$ at $10 \mathrm{~mA} \mathrm{~cm}^{-2}$. Moreover, the new composite also shows a superior bifunctional water splitting performance as catalyst for the OER and HER (hydrogen evolution reaction) catalysts. Our results indicate that the presented material is a promising candidate for water splitting which is cheap and efficient.
\end{abstract}

Keywords: ultrathin structure; oxygen evolution reaction; graphene; two-dimensional; layered double hydroxide

\section{Introduction}

Growing energy consumption and global environmental concerns have attracted lots of interests in seeking clean and sustainable energy sources. In that field, electrocatalytic water splitting has been applied as an effective pathway to realize the conversion of electric energy and the production of hydrogen [1-15]. As a half reaction of the electrocatalytic water splitting, oxygen evolution reaction (OER) is a multi-step four-electron process and shows sluggish kinetics, thus an active catalyst is needed to lower the overpotential of the process [16-18]. Nowadays, noble metals and their oxides based on Ir and Ru are the state-of-art OER catalysts. However, their limited reserve and high price restrict their practical applications [19]. Thus, developing electrocatalysts with high catalytic activity and low price is significantly demanded.

Nowadays, the non-noble metal materials based on $3 \mathrm{~d}$ transition metals, have been widely investigated and became the most potential alternatives for the noble metal catalysts attributed to 
its good availability, low price, environmental friendliness and relatively high activity. Several kinds of catalysts such as transition metal oxides [20-27], hydroxides [3,10,28-31], phosphates [32,33] and sulfides [34-36] have been explored. In addition, the layered double hydroxides (LDH), which are composed of positively charged metal hydroxide layers with intercalated anions, have been regarded as potential catalysts for OER. Compared with unary metal hydroxides, the involvement of two or more transition metals is proved to be beneficial for improving the catalytic activity. The Ni-Fe LDH [10,37], Ni-Co LDH [38], and Ni-V LDH [39] have been investigated and shown better catalytic activity than $\mathrm{Ni}(\mathrm{OH})_{2}$ owing to the novel LDH structure. Furthermore, the ultrathin two-dimensional (2D) structure of LDH also allows it with fast diffusion of ions, which is believed to be important for increasing the OER efficiency [40]. However, the poor electrical conductivity of LDH restricts the proton-coupled electron transfer process and thus limits its practical applications in electrocatalysis.

In order to solve the problem, anchoring the LDH on a conductive substrate, such as carbonaceous materials or metal foams, to boost fast electron transport is considered to be highly effective. As an ideal 2D conductive material, graphene with its atomic thickness, chemical stability, large surface area, and good conductivity has been regarded as an excellent substrate for LDH [41,42]. In this respect, the combination of LDH with highly conductive graphene is expected to realize higher charge-transfer ability and achieve a superior water oxidation performance. Meanwhile, the strategy of integrating of nanosheets on graphene was also reported to tailor the distance between individual nanosheets, prevent the aggregation of the nanosheets and introduce extra interfaces at the interlayer areas to facilitate the electrolyte infiltration into the electrode [43]. These advantages could improve OER performance.

In spite of the electrical conductivity, the active sites of the electrode materials are another significant factor in OER performance. With the development of $2 \mathrm{D}$ ultrathin materials, their advantageous feature such as large specific surface area is beneficial for OER performance. For example, the Fe-Co oxide, Ni-Fe LDH, and Ni-Co LDH ultrathin nanosheets were synthesized and exhibited enhanced OER performances [44-46]. Attributed to the exposure of more active sites on the surface, the ultrathin structure could effectively promote the catalytic reaction. In addition, the low thickness should also reduce the charge transport pathway, thus improving OER performance.

Upon this view, the composite containing active ultrathin LDH and highly conductive graphene is assumed to be an ideal candidate to enhance OER performance. In this work, Mn-Co LDH/graphene composite (denoted as $\mathrm{MnCo}-\mathrm{G}$ ) with ultrathin structure was created for the first time. As expected, the composite achieves a high current density of $461 \mathrm{~mA} \mathrm{~cm}^{-2}$ at $2.0 \mathrm{~V}$ vs. RHE and shows a low overpotential of $0.33 \mathrm{~V}$ at $10 \mathrm{~mA} \mathrm{~cm}^{-2}$. Moreover, the composite shows superior bifunctional water splitting performance as both OER and HER catalysts, thus exhibiting it as a highly active material for OER.

\section{Results and Discussion}

The ultrathin Mn-Co LDH nanosheets (denoted as $\mathrm{MnCo}$ ) were obtained through a convenient room-temperature coprecipitation method. In order to investigate the resulting product, X-ray diffraction (XRD) was done as shown in Figure 1A. The diffraction peaks in the XRD pattern at $12^{\circ}$ and $23^{\circ}$ can be assigned to (003) and (006) facets of the LDH phase. The inset figure shows the crystal structure of the Mn-Co LDH with a typical layered arrangement. Figure 1B represents a transmission electron microscopy (TEM) image showing nanosheet with a size of ca. $100 \mathrm{~nm}$ and a clearly transparent contrast to the substrate can be identified, suggesting the ultrathin nature. Figure $1 \mathrm{C}, \mathrm{D}$ are the high-resolution TEM (HRTEM) images for a nanosheet that an interplanar spacing of $0.17 \mathrm{~nm}$ in the lattice fringes, corresponding to the (012) facets. The selected area electron diffraction (SAED) pattern (inset of Figure 1D) shows a six-fold point pattern, further indicating the phase of Mn-Co LDH. 

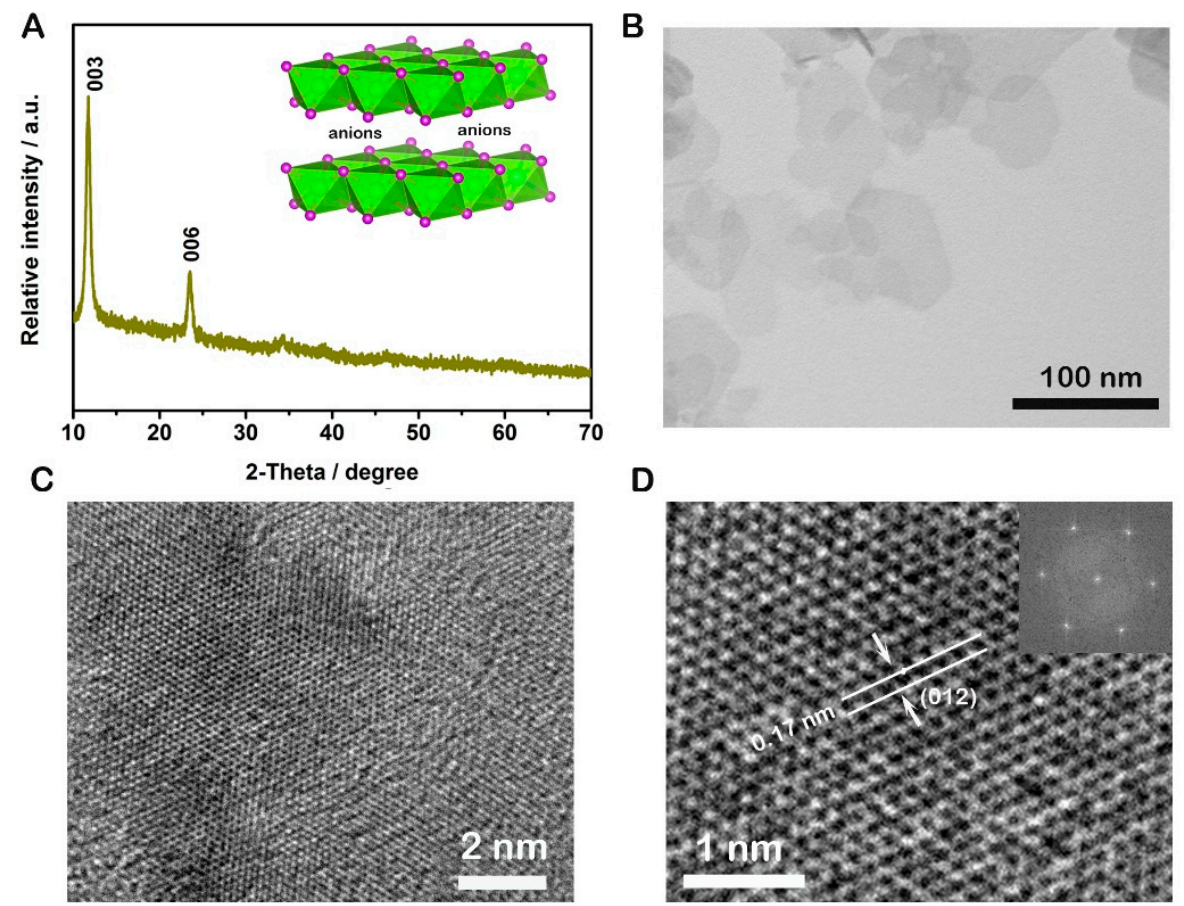

Figure 1. (A) X-ray diffraction (XRD) pattern of the Mn-Co layered double hydroxide (LDH) nanosheets; (B) Transmission electron microscopy (TEM) image of the Mn-Co LDH nanosheets; (C) High-resolution TEM (HRTEM) image of the Mn-Co LDH nanosheets; (D) corresponding selected area electron diffraction (SAED) of the Mn-Co LDH nanosheets.

For the purpose of improving the charge transfer ability of the material, the Mn-Co LDH was combined with the highly conductive graphene. Figure 2A shows the XRD pattern of the obtained composite. Two reflection peaks of the product at $12^{\circ}$ and $23^{\circ}$ can be designated as the layered reflection peaks of $\mathrm{Mn}-\mathrm{Co} \mathrm{LDH}$. It is worthy to note that the peak at $26^{\circ}$ can be assigned to the (002) peak of graphene. In order to confirm the presence of graphene, a Raman spectrum of the product was taken and shown in Figure 2B. Owing to the presence of D band and G band (located at 1347 and $1576 \mathrm{~cm}^{-1}$ ) [47], the graphene component is further confirmed in the composite. The morphology and composition of the sample were further evaluated by the TEM. As shown in Figure 2C, the Mn-Co LDH nanosheets are evenly distributed on the graphene flakes, thus revealing the composite structure of the product. A successful hybridization benefits the electrostatic interaction between the LDH nanosheets and graphene sheets which arise from the residual oxygen-containing functional groups [48]. All these results show that an ultrathin Mn-Co LDH/graphene composite was obtained.
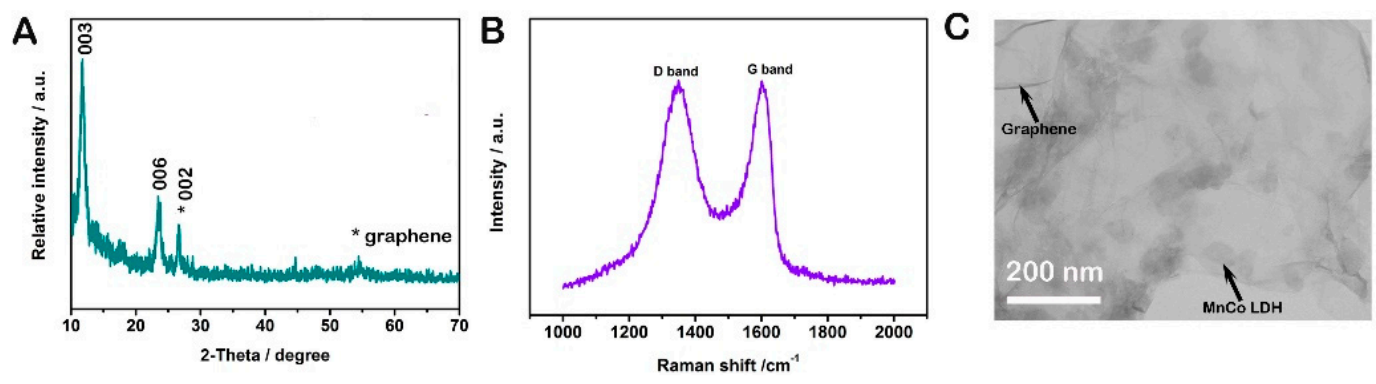

Figure 2. (A) XRD pattern of MnCo-G; (B) Raman spectrum; (C) TEM image of MnCo-G.

For purpose of evaluating the surface structure and chemical valence in the composite, the X-ray photoelectron spectroscopy (XPS) spectra of the product were collected. Figure 3A is the survey 
spectrum, in which typical characteristic peaks of $\mathrm{Mn}, \mathrm{Co}$, and O elements can be recognized. Especially, the spectrum of $\mathrm{Mn} 2 \mathrm{p}$ shows peaks similar to those of $\mathrm{MnOOH}$ and $\mathrm{Mn}_{2} \mathrm{O}_{3}$, indicating the presence of $\mathrm{Mn}^{3+}$ in the product [49]. In Figure 3C, two peak components can be fitted under the Co2 $\mathrm{p}_{3 / 2}$ and $\mathrm{Co} 2 \mathrm{p}_{1 / 2}$, indicating the existence of the $\mathrm{Co}^{2+}$ and $\mathrm{Co}^{3+}$ in the material [50]. Furthermore, Figure 3D shows the $\mathrm{O} 1 \mathrm{~s}$ spectrum which represents the metal-oxygen bonds. The peak located at $531.5 \mathrm{eV}$ is e assigned to the $\mathrm{OH}^{-}$groups [51]. It is worthy to note that $\mathrm{Co}^{3+}$ could be oxidized to $\mathrm{Co}^{4+}$ which is considered as the active sites for OER. In addition, the $\mathrm{Mn}^{3+}$ could also tune the electronic structure of the LDH thus obtained an improved OER performance. The presence of $\mathrm{Co}^{3+}$ and $\mathrm{Mn}^{3+}$ is considered to be beneficial to OER process.
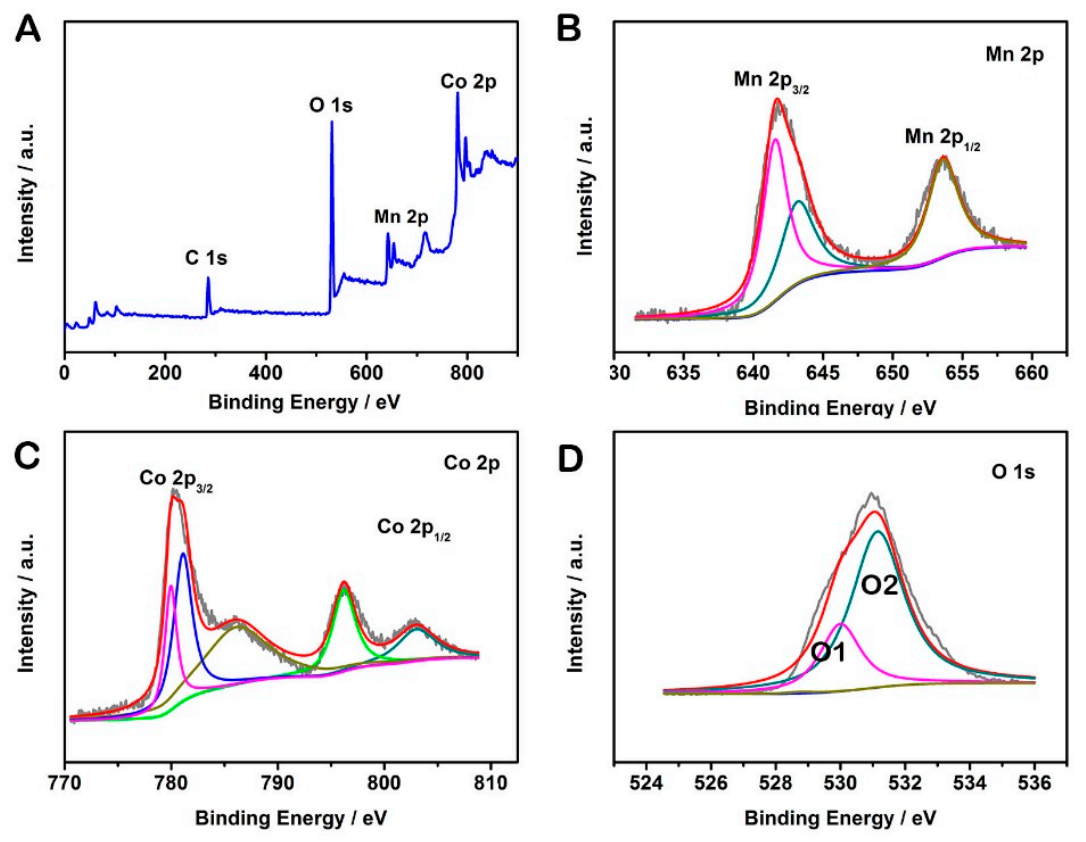

Figure 3. (A) X-ray photoelectron spectroscopy (XPS) survey spectrum, (B) high-resolution XPS spectrum of Mn 2p. (C) Co 2p and (D) O 1s for the MnCo-G sample.

It is worth noting that the composite structure hybridized by catalytically active Mn-Co LDH nanosheet and highly conductive graphene may significantly boost the OER process. In particular, the electroactive Mn-Co LDH nanosheet with ultrathin feature supplies a high surface area, thus increasing the number of active sites number and decreasing the transfer distance of the ions. Furthermore, graphene provides an increased electrical conductivity with a superior charge transfer efficiency. The ultrathin property of the composite could also efficiently reinforce the contact between the catalyst and the substrate [51]. Hence, the composite of Mn-Co LDH/graphene might be an effective catalyst for the water splitting.

For purpose of the investigation for OER performance of the MnCo-G in alkaline solution, the composite was uniformly coated onto a commercial glassy carbon electrode (GCE) for recording linear sweep voltammetry (LSV) curve in a standard three-electrode configuration. In addition, the $\mathrm{MnCo} \mathrm{LDH}$ ultrathin nanosheets without graphene and the benchmarking $\mathrm{RuO}_{2}$ catalyst were also tested as references. Figure 4A and Figure S1 shows the LVS curves of these samples. The Mn-Co $\mathrm{LDH} /$ graphene composite exhibits a small overpotential of $0.33 \mathrm{~V}$ to achieve an OER current density of $10 \mathrm{~mA} \mathrm{~cm}{ }^{-2}$, that value is much lower than the Mn-Co LDH nanosheet without graphene composition. Furthermore, the current density reaches $461 \mathrm{~mA} \mathrm{~cm}^{-2}$ at $2.0 \mathrm{~V}$ for the composite, which is not only higher than the Mn-Co LDH nanosheet but also higher than the benchmarking $\mathrm{RuO}_{2}$. Apart from the low overpotential and high current density, a Tafel slope is used to evaluate the performance of the OER process. By plotting the overpotential versus log current as shown in Figure 4B. The Tafel 
slope of MnCo-G is $48 \mathrm{mV}$ decade ${ }^{-1}$, that is lower than the MnCo LDH nanosheet $\left(63 \mathrm{mV}\right.$ decade $\left.^{-1}\right)$, and even comparable to the noble metal catalyst, $\mathrm{RuO}_{2}\left(42 \mathrm{mV}\right.$ decade $\left.{ }^{-1}\right)$, indicating its facile reaction kinetics for OER catalysis. To prove the durability of $\mathrm{MnCo}-\mathrm{G}$, continuous CV cycling was conducted. As shown in Figure 4C, a negligible degradation of the activity is observed even after $1000 \mathrm{CV}$ cycles, demonstrating the good stability for the MnCo-G composite.

A
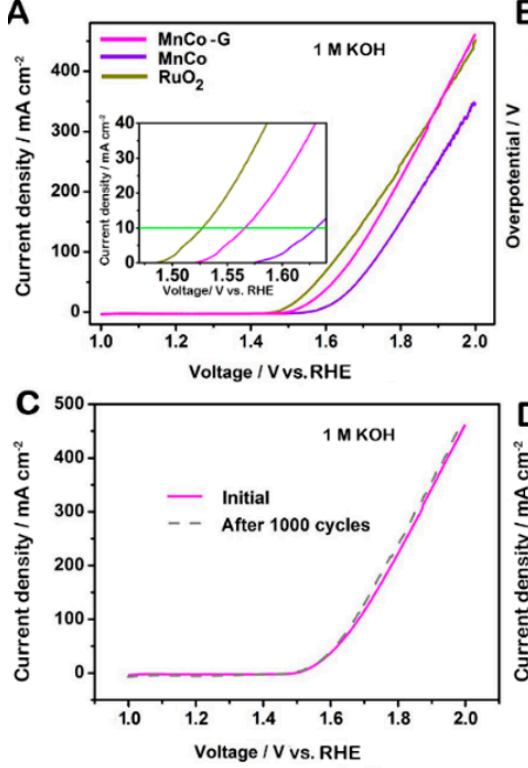

B
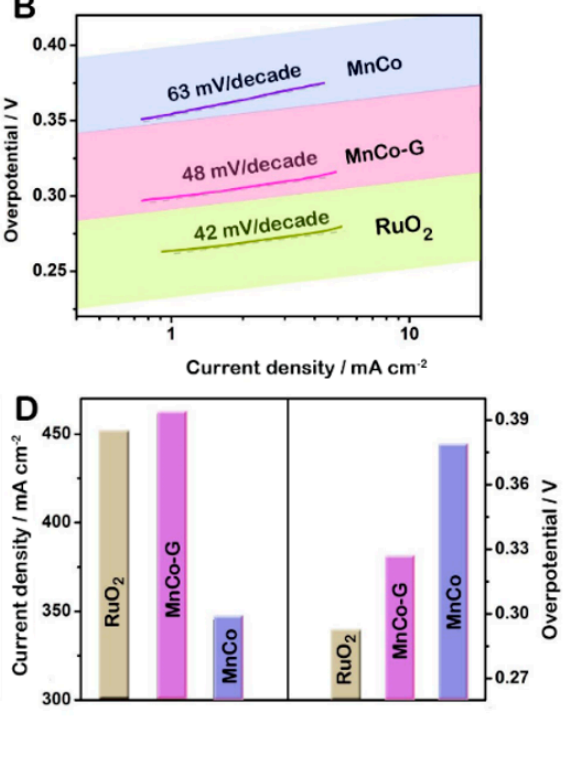

Figure 4. (A) Linear sweep voltammetry (LSV) curves of the catalysts; (B) Corresponding Tafel slopes; (C) LSV curves after $1000 \mathrm{CV}$ cycles for the MnCo-G electrode; (D) comparison of current achieved at the potential of $2.0 \mathrm{~V}$ vs. reversible hydrogen electrode (RHE) and overpotential for $10 \mathrm{~mA} \mathrm{~cm}^{-2}$. All tests were conducted in $1.0 \mathrm{M} \mathrm{KOH}$.

In order to highlight OER performance of the composite, the reported materials based on transition metal oxide and hydroxide were listed in Table 1. As shown, the MnCo-G owns a smaller overpotential and lower Tafel slope than most of the reported materials and is even comparable to the reported $\mathrm{NiFe}-\mathrm{LDH}$, thus confirming its superior OER performance $[33,36,38,52-56]$.

Table 1. Comparison of oxygen evolution reaction (OER) performance with various transition metal oxide and hydroxide.

\begin{tabular}{|c|c|c|c|c|}
\hline Materials & Electrolyte & $\begin{array}{l}\text { Overpotential for } \\
10 \mathrm{~mA} \mathrm{~cm}^{-2} / \mathrm{V}\end{array}$ & Tafel Slope/mV decade ${ }^{-1}$ & Ref. \\
\hline $\mathrm{MnCo}-\mathrm{G}$ & $\mathrm{KOH}$ & 0.33 & 48 & This work \\
\hline $\mathrm{RuO}_{2}$ & $1 \mathrm{M} \mathrm{KOH}$ & 0.3 & 42 & This work \\
\hline $\mathrm{Ni}_{5} \mathrm{Mn}-\mathrm{LDH}-\mathrm{MWCNT}$ & $1 \mathrm{M} \mathrm{KOH}$ & 0.35 (iR-corrected) & 83 & [52] \\
\hline $\mathrm{CO}_{5} \mathrm{Mn}-\mathrm{LDH}-\mathrm{MWCNT}$ & $1 \mathrm{M} \mathrm{KOH}$ & 0.3 (iR-corrected) & 74 & [52] \\
\hline CoNi-LDH/Fe-PP-M & $1 \mathrm{M} \mathrm{KOH}$ & 0.32 & 53 & [53] \\
\hline $\mathrm{CuCo}_{2} \mathrm{O}_{4} / \mathrm{N}-\mathrm{rGO}$ & $1 \mathrm{M} \mathrm{KOH}$ & 0.36 & 64 & [54] \\
\hline $\mathrm{Co}_{3} \mathrm{~S}_{4} @ \mathrm{MoS}_{2}$ & $1 \mathrm{M} \mathrm{KOH}$ & 0.33 & 59 & [36] \\
\hline $\mathrm{CoMoO}_{4}$ & $1 \mathrm{M} \mathrm{KOH}$ & 0.31 & 56 & {$[55]$} \\
\hline $\mathrm{CoP}$ & $1 \mathrm{M} \mathrm{KOH}$ & 0.36 & 66 & [33] \\
\hline CoFe LDH & $0.1 \mathrm{M} \mathrm{KOH}$ & 0.36 & 49 & [56] \\
\hline $\mathrm{NiFe} \mathrm{LDH}$ & $1 \mathrm{M} \mathrm{KOH}$ & 0.33 & 41 & [38] \\
\hline
\end{tabular}

Inspired by the superior OER catalytic activity, the catalytic performance for complete water splitting process was investigated by loading MnCo-G and MnCo onto a nickel foam (NF) substrate. 
As depicted in Figure 5A,B, the MnCo-G/NF shows a higher current density and lower onset overpotential for both the OER and HER processes. Moreover, a two-electrode system with $\mathrm{MnCo}-\mathrm{G} / \mathrm{NF}$ used as anode and cathode was tested in $1 \mathrm{M} \mathrm{KOH}$ electrolyte. In Figure 5C, the MnCo-G sample shows a lower applied voltage at $60 \mathrm{~mA} \mathrm{~cm}^{-2}$ compared to the MnCo sample. The current density was very steady and maintained for at least $22 \mathrm{~h}$ (Figure S2). These data undoubtedly show the excellent water splitting performance of the obtained MnCo-G sample.
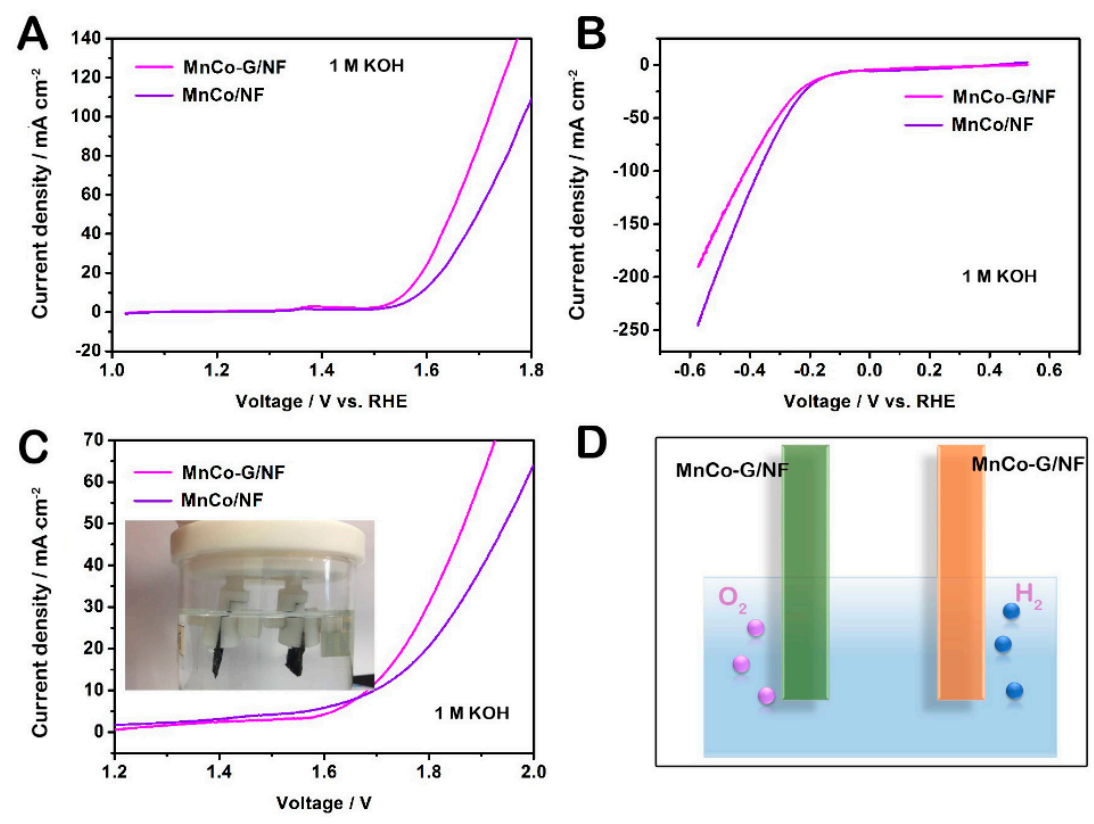

Figure 5. (A) OER and (B) HER polarization curves of MnCo-G and MnCo catalysts loaded onto nickel foam substrate; (C) full water splitting characteristics in a two-electrode configuration for the two samples; (D) schematic image for the full water splitting process.

In order to investigate the factors that influence the catalytic activity, the MnCo-G and MnCo LDH samples were loaded onto GCE to evaluate the electrochemical double-layer capacitance $\left(C_{\mathrm{dl}}\right)$ in Figure 6A,B. The higher $\mathrm{Cdl}$ value represents the lager electrochemically active surface area (ECSA) of the sample [57-59]. In Figure 6A and Figure S3, a current density from 1 to $9 \mathrm{mV} \mathrm{s}^{-1}$ was measured in a non-Faradic region, which can be plotted as a function of the sweep rate to evaluate the $C_{\mathrm{dl}}$. As can be seen, the MnCo-G electrode exhibits a higher $\mathrm{C}_{\mathrm{dl}}$ of $0.23 \mathrm{mF} \mathrm{cm}{ }^{-2}$ than the MnCo electrode with $0.18 \mathrm{mF} \mathrm{cm}^{-2}$, thus revealing an increased active area of the MnCo-G sample. This is attributed to the introduction of graphene which prevents the aggregation of the ultrathin nanosheets and increases the overall conductivity of the product, thus leading to an increased specific surface area. Furthermore, a larger $C_{\mathrm{dl}}$ means more ion accessible surface area and surface active sites for electrolyte permeation, which would enhance the electrocatalytic process. Furthermore, catalytic kinetics of MnCo and MnCo-G were estimated with electrochemical impedance spectroscopy (EIS, Figure 6C) to clarify the role of graphene. Compared to $\mathrm{MnCo}$, the $\mathrm{MnCo}-\mathrm{G}$ catalyst shows a smaller semicircle in the EIS which demonstrates a faster charge transfer. The impedance results obtained by fitting the equivalent circuit (Figure $6 \mathrm{E}$ ) are tabulated in Figure $6 \mathrm{D}$. The $\mathrm{C}_{\mathrm{dl}}$ is the double-layer capacitance and $R_{s}$ is the solution resistance. The $R_{p}$ and $R_{3}$ are corresponding to the kinetics of the charge transfer process. The polarization resistance $R_{p}$ is an indicator of the catalytic performance of the OER [52]. As can be seen, the MnCo-G composite shows lower $R_{p}$ and $R_{3}$ thus indicating a higher charge transfer capability. This is due to the introduction of graphene which provides a high electrical conductivity thus accelerating the charge transport during the OER process. All the above data 
confirm the advantageous composite structure of electroactive Mn-Co LDH and electrically conductive graphene in the water oxidation application.
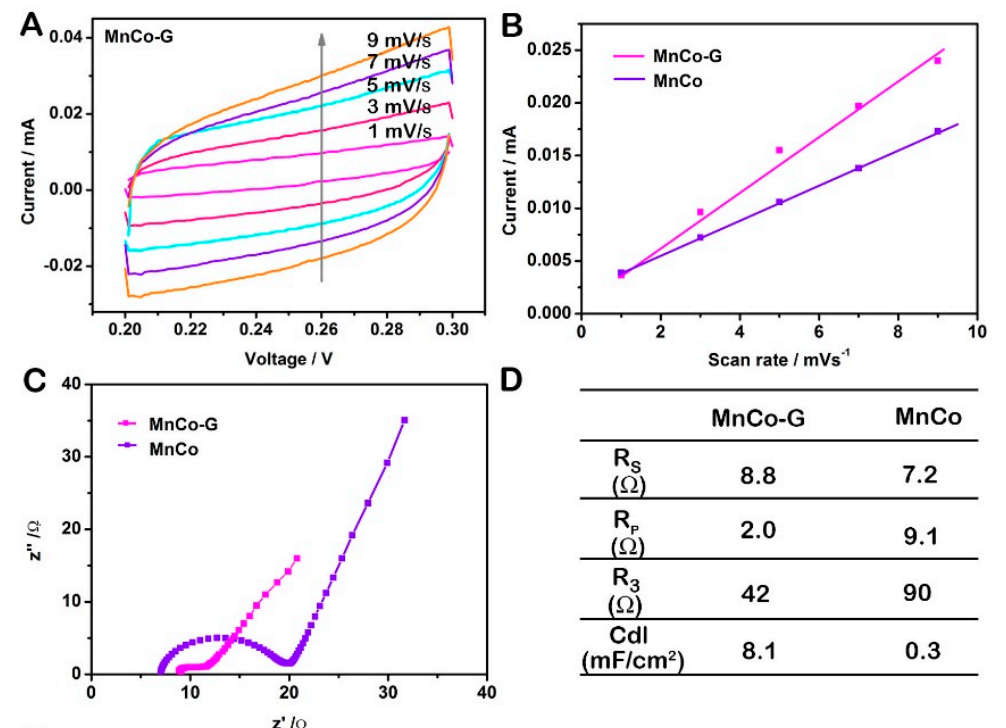

\begin{tabular}{ccc}
\hline & MnCo-G & MnCo \\
\hline $\begin{array}{c}\mathrm{R}_{\mathrm{S}} \\
(\Omega)\end{array}$ & 8.8 & 7.2 \\
\hline $\begin{array}{c}\mathrm{R}_{\mathrm{p}} \\
(\Omega)\end{array}$ & 2.0 & 9.1 \\
\hline $\begin{array}{c}\mathrm{R}_{3} \\
(\Omega)\end{array}$ & 42 & 90 \\
\hline $\begin{array}{c}\mathrm{Cdl} \\
\left(\mathrm{mF} / \mathrm{cm}^{2}\right)\end{array}$ & 8.1 & 0.3 \\
\hline
\end{tabular}

E

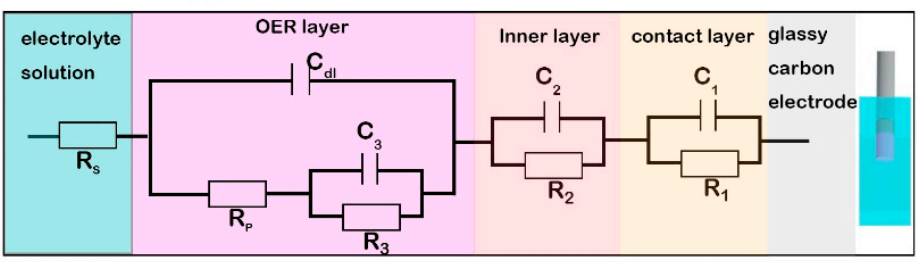

Figure 6. (A) CVs of the Mn-Co LDH/graphene loaded onto GCE and measured at scan rates from 1 to $9 \mathrm{mV} \mathrm{s}^{-1}$; (B) $\mathrm{C}_{\mathrm{dl}}$ plots for the MnCo-G and MnCo sample; (C) Nyquist plots of Mn-Co LDH/graphene electrode and Mn-Co LDH; (D) summary of the fitted values of $\mathrm{C}_{\mathrm{dl}}, \mathrm{R}_{\mathrm{s}}, \mathrm{R}_{3}$, and $\mathrm{R}_{\mathrm{p}} ;(\mathrm{E})$ the equivalent circuit for modeling impedance data. All the tests were conducted in $1 \mathrm{M} \mathrm{KOH}$.

The structural advantage based on ultrathin Mn-Co LDH nanosheets integrated on graphene sheets is summarized as follows. Firstly, graphene can serve as a highly conductive substrate for ultrathin Mn-Co LDH nanosheets, thus leading to an improved electrical conductivity boosting the charge transfer process. Meanwhile, the Mn-Co LDH integrated on graphene can potentially tailor the distance between individual nanosheets, prevent the aggregation of the nanosheets, and introduce extra interfaces at the interlayer areas to facilitate the electrolyte infiltration into the catalyst during the water splitting processes. Furthermore, owing to the high exposure of the catalytically active surface metal atoms, the ultrathin structure could also effectively promote the catalytic reaction. Altogether, the Mn-Co LDH/graphene composite is an effective catalyst for water oxidation.

\section{Experimental Section}

\subsection{Syntheses of the Mn-Co LDH/Graphene Ultrathin Composition Structure}

All the chemicals were purchased from Sinopharm Chemical Reagent Co. Ltd. (Shanghai, China) Typically, $0.135 \mathrm{~g} \mathrm{Co}\left(\mathrm{NO}_{3}\right)_{2} \cdot 6 \mathrm{H}_{2} \mathrm{O}, 0.005 \mathrm{~g} \mathrm{Mn}\left(\mathrm{NO}_{3}\right)_{2} \cdot 4 \mathrm{H}_{2} \mathrm{O}, 0.07 \mathrm{~g} \mathrm{NaNO}_{3}$ and $0.09 \mathrm{~g} \mathrm{NH}_{4} \mathrm{~F}$ were dissolved in $100 \mathrm{~mL} \mathrm{H}_{2} \mathrm{O}$ under $\mathrm{N}_{2}$ protection at room temperature. Then $12.5 \mu \mathrm{L} \mathrm{H}_{2} \mathrm{O}_{2}$ (30 wt \%) was dropped into the solution with vigorous stirring for $30 \mathrm{~min}$. The addition of $35 \mathrm{~mL} \mathrm{NaOH}$ solution $(0.08 \mathrm{M})$ was used to adjust the $\mathrm{pH}$ of the solution to 10 . The obtained suspension was aged overnight. Then it was isolated by centrifugation, washed with $\mathrm{H}_{2} \mathrm{O}$ and anhydrous ethanol, and dried in ambient conditions. The obtained LDH material was dispersed into the $\mathrm{N}_{2}$ saturated solution contained $1 \mathrm{M} \mathrm{NaCl}$ and $3.3 \mathrm{mM} \mathrm{HCl}$, after vigorous stirring, the suspension was washed with water and ethanol 
(saturated with $\mathrm{N}_{2}$ ). $10 \mathrm{mg}$ of graphene (Ningbo Institute of Industrial Technology, Ningbo, China) raw solution was added to $20 \mathrm{~mL}$ of N,N-Dimethylformamide (DMF) with ultrasonication for $12 \mathrm{~h}$. After that, the dispersion was centrifuged with a low speed of $1000 \mathrm{rpm}$ to get the exfoliated graphene. Then, $10 \mathrm{mg}$ of LDH was dispersed into the deionized water and $2 \mathrm{~mL}$ of graphene suspension was added into the dispersion agent. After the ultrasonication and the following magnetic stirring, the suspension was collected, washed with water and dried in the vacuum oven. The content of graphene in the composite is about $9 \mathrm{wt} \%$.

\subsection{Materials Characterizations}

The XRD measurements were taken on a Philips X'Pert Pro Super diffractometer (Amsterdam, The Netherlands) with $\mathrm{Cu} \mathrm{K} \alpha$ radiation $(\lambda=0.154178 \mathrm{~nm})$. The HRTEM and corresponding SAED were taken at a JEOL-2010 (JEOL, Tokyo, Japan) operated at $200 \mathrm{kV}$. The Raman spectrum was tested at a LABRAM-HR (Horiba-Jobin Yvon, Edison, NJ, USA) Confocal laser Raman spectrometer. The X-ray photoelectron spectrometry (XPS) was performed on an ESCALAB 250Xi Instrument (ThermoFisher Scientific, Waltham, MA, USA).

\subsection{Electrochemical Tests}

The electrochemical tests were conducted in the three-electrode configuration using CHI660B potentiostat (Shanghai Chenhua Instrumental Co. Ltd., Shanghai, China). An Ag/ AgCl electrode was taken as the reference electrode. $4 \mathrm{mg}$ of catalyst and $25 \mu \mathrm{L}$ Nafion solution (Sigma Aldrich, St. Louis, MO, USA) were dissolved in a water/isopropanol solution ( $1 \mathrm{~mL}, 3: 1 v / v)$. Then, the solution was further sonicated for $30 \mathrm{~min}$. After that, $5 \mu \mathrm{L}$ of the catalyst ink suspension was coated onto a commercial glassy carbon electrode (GCE, $3 \mathrm{~mm}$ diameter, $0.07 \mathrm{~cm}^{2}$ ) with a mass loading of $0.285 \mathrm{mg} \mathrm{cm}^{-2}$ and dried at room temperature. Then, the OER test was conducted in $\mathrm{N}_{2}$-saturated $1 \mathrm{M} \mathrm{KOH}$ and $0.1 \mathrm{M} \mathrm{KOH}$ solution. LSV curve with a scan rate of $5 \mathrm{mV} \mathrm{s}^{-1}$ was carried out in $1 \mathrm{M}$ and $0.1 \mathrm{M} \mathrm{KOH}$. CV was done in the voltage range of 0 to $1.0 \mathrm{~V} \mathrm{vs}$. $\mathrm{Ag} / \mathrm{AgCl}$ at $5 \mathrm{mV} \mathrm{s}^{-1}$ to test cycling stability. The EIS was done in the frequency range from $100 \mathrm{kHz}$ to $1 \mathrm{~Hz}$ at an amplitude voltage of $5 \mathrm{mV}$. For overall water splitting, the catalysts were loaded onto the nickel foam substrate $\left(2 \mathrm{mg} \mathrm{cm}^{-2}\right)$ and tested in the $1 \mathrm{M} \mathrm{KOH}$ solution, with a Pt plate and carbon rod as the counter electrode for OER and HER test, respectively. The corresponding characterizations and properties of graphene and nickel foam are shown in Figures S4-S7 and Table S1.

\section{Conclusions}

Mn-Co LDH/graphene composite was synthesized for the first time in order to solve the problem of the low electrical conductivity of Mn-Co LDH. Due to the enhanced charge transfer ability, the Mn-Co $\mathrm{LDH} /$ graphene composite shows an excellent OER performance. As the OER catalyst, the composite achieves a high current density of $461 \mathrm{~mA} \mathrm{~cm}^{-2}$ at $2.0 \mathrm{~V}$ and require a low overpotential of $0.33 \mathrm{~V}$ to reach an OER current density of $10 \mathrm{~mA} \mathrm{~cm}^{-2}$. These data are not only better than the conventional electrode materials based on the transition metal oxides and hydroxides but also comparable to the state-of-the-art $\mathrm{RuO}_{2}$. By the way, the composite also shows superior full water splitting performance when used as a bifunctional catalyst. In the end, this work reveals the promising catalytic properties of $\mathrm{Mn}-\mathrm{Co} \mathrm{LDH} /$ graphene composite material and expands the scope of non-noble metal catalysts with an excellent performance for the water oxidation reaction.

Supplementary Materials: The following are available online at http:/ /www.mdpi.com/2073-4344/8/9/350/s1. Figure S1: LSV curves in $0.1 \mathrm{M} \mathrm{KOH}$ solution, Figure S2: I-t curve for the overall water splitting process, Figure S3: CVs of the Mn-Co LDH electrode measured in $1 \mathrm{M} \mathrm{KOH}$ at scan rates from 1 to $9 \mathrm{mV} \mathrm{s}^{-1}$, Figure S4: XRD patterns of nickel foam (A) and graphene (B), Figure S5: The LSV curves of NF for the OER (A), HER (B) and overall water splitting (C), Figure S6: The LSV curve of graphene for the OER process, Figure S7: CVs of the graphene electrode measured in $1 \mathrm{M} \mathrm{KOH}$ at scan rates from 1 to $9 \mathrm{mV} \mathrm{s}^{-1}$, (B) Current density as a function of scan rate for the graphene. (C) Nyquist plots of graphene electrode in 0.6 V, Table S1: The ICP results in graphene, nickel foam and electrolyte after stability test. 
Author Contributions: J.B., J.X. and H.L. designed the experiments and wrote the paper; Z.W. and W.L. performed the experiments; L.X. and M.G. analyzed the data; F.L. and Y.Z. produced the graphs and figures.

Acknowledgments: This work was financially supported by the National Natural Science Foundation of China for Youths (No. 21601067, 21501112, 21506081, 51603092), the Natural Science Foundation of the Jiangsu Province for Youths (No. BK20160492), the Natural Science Foundation of Shandong Province (No. ZR2018JL009), the University Natural Science Research of Jiangsu (No. 16KJB150008), the Jiangsu Province Postdoctoral Science Foundation (No. 1601253C), the China Postdoctoral Science Foundation (No. 2016M590415), the Funding for scientific research startup of Jiangsu University (No. 15JDG161) and a Project Funded by the Priority Academic Program Development of the Jiangsu Higher Education Institutions.

Conflicts of Interest: The authors declare no conflict of interest.

\section{References}

1. Xie, J.; Xie, Y. Structural Engineering of Electrocatalysts for the Hydrogen Evolution Reaction: Order or Disorder? ChemCatChem 2015, 7, 2568-2580. [CrossRef]

2. Xie, J.; Xie, Y. Transition metal nitrides for electrocatalytic energy conversion: opportunities and challenges. Chem. Eur. J. 2015, 22, 3588-3598. [CrossRef] [PubMed]

3. Jin, Y.; Huang, S.; Yue, X.; Du, H.; Shen, P.K. Mo and Fe Modified Ni(OH $)_{2} / \mathrm{NiOOH}$ Nanosheets as highly active and stable electrocatalysts for oxygen evolution reaction. ACS Catal. 2018, 8, 2359-2363. [CrossRef]

4. Long, X.; Wang, Z.; Xiao, S.; An, Y.; Yang, S. Transition metal based layered double hydroxides tailored for energy conversion and storage. Mater. Today 2016, 19, 213-226. [CrossRef]

5. Xie, J.; Liu, W.; Xin, J.; Lei, F.; Gao, L.; Qu, H.; Zhang, X.; Xie, Y. Dual effect in fluorine-doped hematite nanocrystals for efficient water oxidation. ChemSusChem 2017, 10, 4465-4471. [CrossRef] [PubMed]

6. Chen, S.; Kang, Z.; Zhang, X.; Xie, J.; Wang, H.; Shao, W.; Zheng, X.S.; Yan, W.; Pan, B.; Xie, Y. Highly active $\mathrm{Fe}$ sites in ultrathin pyrrhotite $\mathrm{Fe}_{7} \mathrm{~S}_{8}$ nanosheets realizing efficient electrocatalytic oxygen evolution. ACS Cent. Sci. 2017, 3, 1221-1227. [CrossRef] [PubMed]

7. Xie, J.; Liu, W.; Lei, F.; Zhang, X.; Qu, H.; Gao, L.; Hao, P.; Tang, B.; Xie, Y. Iron-Incorporated $\alpha-\mathrm{Ni}(\mathrm{OH})_{2}$ Hierarchical Nanosheet Arrays for Electrocatalytic Urea Oxidation. Chem. Eur. J. 2018. [CrossRef] [PubMed]

8. Xie, J.; Wang, R.; Bao, J.; Zhang, X.; Zhang, H.; Li, S.; Xie, Y. Zirconium trisulfide ultrathin nanosheets as efficient catalysts for water oxidation in both alkaline and neutral solutions. Inorg. Chem. Front. 2014, 1, 751-756. [CrossRef]

9. Zhao, Y.; Zhang, X.; Jia, X.; Waterhouse, G.I.N.; Shi, R.; Zhang, X.; Zhan, F.; Tao, Y.; Wu, L.; Tung, C.; et al. Sub-3 nm ultrafine monolayer layered double hydroxide nanosheets for electrochemical water oxidation. Adv. Energy Mater. 2018, 8, 1703585. [CrossRef]

10. Wang, F.F.; Wang, T.; Sun, S.G.; Xu, Y.Q.; Yu, R.J.; Li, H.J. One-step synthesis of nickle iron-layered double hydroxide/reduced graphene oxide/carbon nanofibres composite as electrode materials for asymmetric supercapacitor. Sci. Rep. 2018, 8, 8908. [CrossRef] [PubMed]

11. Liardet, L.; Hu, X. Amorphous cobalt vanadium oxide as a highly active electrocatalyst for oxygen evolution. ACS Catal. 2017, 8, 644-650. [CrossRef] [PubMed]

12. Xie, J.; Qu, H.; Xin, J.; Zhang, X.; Cui, G.; Zhang, X.; Bao, J.; Tang, B.; Xie, Y. Defect-rich MoS 2 nanowall catalyst for efficient hydrogen evolution reaction. Nano Res. 2017, 10, 1178-1188. [CrossRef]

13. Xie, J.; Zhang, H.; Li, S.; Wang, R.; Sun, X.; Zhou, M.; Zhou, J.; Lou, X.W.D.; Xie, Y. Defect-rich MoS 2 ultrathin nanosheets with additional active edge sites for enhanced electrocatalytic hydrogen evolution. Adv. Mater. 2013, 25, 5807-5813. [CrossRef] [PubMed]

14. Masudy-Panah, S.; Siavash Moakhar, R.; Chua, C.S.; Tan, H.R.; Wong, T.I.; Chi, D.; Dalapati, G.K. Nanocrystal engineering of sputter-grown $\mathrm{CuO}$ photocathode for visible-light-driven electrochemical water splitting. ACS Appl. Mater. Interfaces 2016, 8, 1206-1213. [CrossRef] [PubMed]

15. Xie, J.; Xin, J.; Cui, G.; Zhang, X.; Zhou, L.; Wang, Y.; Liu, W.; Wang, C.; Ning, M.; Xia, X.; et al. Vertically aligned oxygen-doped molybdenum disulfide nanosheets grown on carbon cloth realizing robust hydrogen evolution reaction. Inorg. Chem. Front. 2016, 3, 1160-1166. [CrossRef]

16. Bao, J.; Wang, Z.; Liu, W.; Xu, L.; Lei, F.; Xie, J.; Zhao, Y.; Huang, Y.; Guan, M.; Li, H. ZnCo ${ }_{2} \mathrm{O}_{4}$ Ultrathin nanosheets towards the high performance of flexible supercapacitors and bifunctional electrocatalysis. J. Alloys Compd. 2018, 764, 565-573. [CrossRef] 
17. Xie, J.; Zhang, X.; Zhang, H.; Zhang, J.; Li, S.; Wang, R.; Pan, B.; Xie, Y. Intralayered ostwald ripening to ultrathin nanomesh catalyst with robust oxygen-evolving performance. Adv. Mater. 2017, 29, 1604765. [CrossRef] [PubMed]

18. Lee, Y.; Suntivich, J.; May, K.J.; Perry, E.E.; Shao-Horn, Y. Synthesis and activities of rutile $\mathrm{IrO}_{2} \mathrm{and}_{\mathrm{RuO}}$ nanoparticles for oxygen evolution in acid and alkaline solutions. J. Phys. Chem. Lett. 2012, 399-404. [CrossRef] [PubMed]

19. Aijaz, A.; Masa, J.; Rösler, C.; Xia, W.; Weide, P.; Botz, A.J.R.; Fischer, R.A.; Schuhmann, W.; Muhler, M. $\mathrm{Co} @ \mathrm{Co}_{3} \mathrm{O}_{4}$ encapsulated in carbon nanotube-grafted nitrogen-doped carbon polyhedra as an advanced bifunctional oxygen electrode. Angew. Chem. Int. Ed. 2016, 55, 4087-4091. [CrossRef] [PubMed]

20. Liang, Y.; Li, Y.; Wang, H.; Zhou, J.; Wang, J.; Regier, T.; Dai, $\mathrm{H} . \mathrm{Co}_{3} \mathrm{O}_{4}$ nanocrystals on graphene as a synergistic catalyst for oxygen reduction reaction. Nat. Mater. 2011, 10, 780-786. [CrossRef] [PubMed]

21. Yeo, B.S.; Bell, A.T. Enhanced activity of gold-supported cobalt oxide for the electrochemical evolution of oxygen. J. Am. Chem. Soc. 2011, 133, 5587-5593. [CrossRef] [PubMed]

22. Li, Y.; Hasin, P.; $\mathrm{Wu}, \mathrm{Y} . \mathrm{Ni}_{\mathrm{x}} \mathrm{Co}_{3-\mathrm{x}} \mathrm{O}_{4}$ nanowire arrays for electrocatalytic oxygen evolution. Adv. Mater. 2010, 22, 1926-1929. [CrossRef] [PubMed]

23. Ramírez, A.; Bogdanoff, P.; Friedrich, D.; Fiechter, S. Synthesis of $\mathrm{Ca}_{2} \mathrm{Mn}_{3} \mathrm{O}_{8}$ films and their electrochemical studies for the oxygen evolution reaction (OER) of water. Nano Energy 2012, 1, 282-289. [CrossRef]

24. Prabu, M.; Ketpang, K.; Shanmugam, S. Hierarchical nanostructured $\mathrm{NiCO}_{2} \mathrm{O}_{4}$ as an efficient bifunctional non-precious metal catalyst for rechargeable zinc-air batteries. Nanoscale 2014, 6, 3173. [CrossRef] [PubMed]

25. Manivasakan, P.; Ramasamy, P.; Kim, J. Use of urchin-like $\mathrm{Ni}_{\mathrm{x}} \mathrm{Co}_{3-\mathrm{x}} \mathrm{O}_{4}$ hierarchical nanostructures based on non-precious metals as bifunctional electrocatalysts for anion-exchange membrane alkaline alcohol fuel cells. Nanoscale 2014, 6, 9665-9672. [CrossRef] [PubMed]

26. Mondschein, J.S.; Callejas, J.F.; Read, C.G.; Chen, J.Y.C.; Holder, C.F.; Badding, C.K.; Schaak, R.E. Crystalline cobalt oxide films for sustained electrocatalytic oxygen evolution under strongly acidic conditions. Chem. Mater. 2017, 29, 950-957. [CrossRef]

27. Lübke, M.; Sumboja, A.; McCafferty, L.; Armer, C.F.; Handoko, A.D.; Du, Y.; McColl, K.; Cora, F.; Brett, D.; $\mathrm{Liu}, \mathrm{Z}$; et al. Transition-metal-doped $\alpha-\mathrm{MnO}_{2}$ nanorods as bifunctional catalysts for efficient oxygen reduction and evolution reactions. Chem. Select. 2018, 9, 2613-2622. [CrossRef]

28. Xie, J.; Xin, J.; Wang, R.; Zhang, X.; Lei, F.; Qu, H.; Hao, P.; Cui, G.; Tang, B.; Xie, Y. Sub-3 nm pores in two-dimensional nanomesh promoting the generation of electroactive phase for robust water oxidation. Nano Energy 2018, 53, 74-82. [CrossRef]

29. Wang, L.; Lin, C.; Huang, D.; Zhang, F.; Wang, M.; Jin, J. A comparative study of composition and morphology effect of $\mathrm{Ni}_{\mathrm{x}} \mathrm{Co}_{1-\mathrm{x}}(\mathrm{OH})_{2}$ on oxygen evolution/reduction reaction. ACS Appl. Mater. Interfaces 2014, 6, 10172-10180. [CrossRef] [PubMed]

30. Zhao, Z.; Wu, H.; He, H.; Xu, X.; Jin, Y. A high-performance binary Ni-CO hydroxide-based water oxidation electrode with three-dimensional coaxial nanotube array structure. Adv. Funct. Mater. 2014, 24, 4698-4705. [CrossRef]

31. Gao, M.; Sheng, W.; Zhuang, Z.; Fang, Q.; Gu, S.; Jiang, J.; Yan, Y. Efficient water oxidation using nanostructured $\alpha$-nickel-hydroxide as an electrocatalyst. J. Am. Chem. Soc. 2014, 136, 7077-7084. [CrossRef] [PubMed]

32. Kanan, M.W.; Yano, J.; Surendranath, Y.; Dincă, M.; Yachandra, V.K.; Nocera, D.G. Structure and valency of a cobalt-phosphate water oxidation catalyst determined by in situ X-ray spectroscopy. J. Am. Chem. Soc. 2010, 132, 13692-13701. [CrossRef] [PubMed]

33. Ryu, J.; Jung, N.; Jang, J.H.; Kim, H.-J.; Yoo, S.J. In Situ transformation of hydrogen-evolving CoP nanoparticles: Toward efficient oxygen evolution catalysts bearing dispersed morphologies with Co-oxo/hydroxo molecular units. ACS Catal. 2015, 5, 4066-4074. [CrossRef]

34. Bediako, D.K.; Surendranath, Y.; Nocera, D.G. Mechanistic studies of the oxygen evolution reaction mediated by a nickel-borate thin film electrocatalyst. J. Am. Chem. Soc. 2013, 135, 3662-3674. [CrossRef] [PubMed]

35. Gao, M.-R.; Cao, X.; Gao, Q.; Xu, Y.-F.; Zheng, Y.-R.; Jiang, J.; Yu, S.-H. Nitrogen-doped graphene supported $\mathrm{CoSe}_{2}$ nanobelt composite catalyst for efficient water oxidation. ACS Nano 2014, 8, 3970-3978. [CrossRef] [PubMed] 
36. Guo, Y.; Tang, J.; Qian, H.; Wang, Z.; Yamauchi, Y. One-Pot synthesis of zeolitic imidazolate framework 67-derived hollow $\mathrm{Co}_{3} \mathrm{~S}_{4} @ \mathrm{MoS}_{2}$ heterostructures as efficient bifunctional catalysts. Chem. Mater. 2017, 29, 5566-5573. [CrossRef]

37. Lu, Z.; Xu, W.; Zhu, W.; Yang, Q.; Lei, X.; Liu, J.; Li, Y.; Sun, X.; Duan, X. Three-dimensional NiFe layered double hydroxide film for high-efficiency oxygen evolution reaction. Chem. Commun. 2014, 50, 6479-6482. [CrossRef] [PubMed]

38. Song, F.; Hu, X. Exfoliation of layered double hydroxides for enhanced oxygen evolution catalysis. Nat. Commun. 2014, 5, 4477. [CrossRef] [PubMed]

39. Fan, K.; Chen, H.; Ji, Y.; Huang, H.; Claesson, P.M.; Daniel, Q.; Philippe, B.; Rensmo, H.; Li, F.; Luo, Y.; et al. Nickel-vanadium monolayer double hydroxide for efficient electrochemical water oxidation. Nat. Commun. 2016, 7, 11981. [CrossRef] [PubMed]

40. Song, F.; Hu, X. Ultrathin cobalt-manganese layered double hydroxide is an efficient oxygen evolution catalyst. J. Am. Chem. Soc. 2014, 136, 16481-16484. [CrossRef] [PubMed]

41. Kumar, R.K.; Bandurin, D.A.; Pellegrino, F.M.D.; Cao, Y.; Principi, A.; Guo, H.; Auton, G.H.; Shalom, M.B.; Ponomarenko, L.A.; Falkovich, G.; et al. Superballistic flow of viscous electron fluid through graphene constrictions. Nat. Phys. 2017, 13, 1182-1185. [CrossRef]

42. Sanchez-Yamagishi, J.D.; Luo, J.Y.; Young, A.F.; Hunt, B.M.; Watanabe, K.; Taniguchi, T.; Ashoori, R.C.; Jarillo-Herrero, P. Helical edge states and fractional quantum hall effect in a graphene electron-hole bilayer. Nat. Nanotechnol. 2017, 12, 118-122. [CrossRef] [PubMed]

43. Peng, L.; Peng, X.; Liu, B.; Wu, C.; Xie, Y.; Yu, G. Ultrathin two-dimensional $\mathrm{MnO}_{2}$ /graphene hybrid nanostructures for high-performance, flexible planar supercapacitors. Nano Lett. 2013, 13, 2151-2157. [CrossRef] [PubMed]

44. Zhuang, L.; Ge, L.; Yang, Y.; Li, M.; Jia, Y.; Yao, X.; Zhu, Z. Ultrathin iron-cobalt oxide nanosheets with abundant oxygen vacancies for the oxygen evolution reaction. Adv. Mater. 2017, 29, 1606793. [CrossRef] [PubMed]

45. Yu, L.; Yang, J.F.; Guan, B.Y.; Lu, Y.; Lou, X.W.D. Hierarchical hollow nanoprisms based on ultrathin Ni-Fe layered double hydroxide nanosheets with enhanced electrocatalytic activity towards oxygen evolution. Angew. Chem. Int. Ed. 2018, 57, 172-176. [CrossRef] [PubMed]

46. Wang, Y.; Xie, C.; Zhang, Z.; Liu, D.; Chen, R.; Wang, S. In Situ exfoliated, N-doped, and edge-rich ultrathin layered double hydroxides nanosheets for oxygen evolution reaction. Adv. Funct. Mater. 2018, 28, 1703363. [CrossRef]

47. Wu, J.-B.; Lin, M.-L.; Cong, X.; Liu, H.-N.; Tan, P.H. Raman spectroscopy of graphene-based materials and its applications in related devices. Chem. Soc. Rev. 2018, 47, 1822-1873. [CrossRef] [PubMed]

48. Xie, J.; Sun, X.; Zhang, N.; Xu, K.; Zhou, M.; Xie, Y. Layer-by-layer $\beta-\mathrm{Ni}(\mathrm{OH})_{2} /$ graphene nanohybrids for ultraflexible all-solid-state thin-film supercapacitors with high electrochemical performance. Nano Energy 2013, 2, 65-74. [CrossRef]

49. Biesinger, M.C.; Payne, B.P.; Grosvenor, A.P.; Lau, L.W.M.; Gerson, A.R.; Smart, R.S.C. Resolving surface chemical states inXPS analysis of first row transition metals, oxides and hydroxides: $\mathrm{Cr}, \mathrm{Mn}, \mathrm{Fe}, \mathrm{Co}$ and $\mathrm{Ni}$. Appl. Surf. Sci. 2011, 257, 2717-2730. [CrossRef]

50. Li, J.; Wang, J.; Wexler, D.; Shi, D.; Liang, J.; Liu, H.; Xiong, S.; Qian, Y. Simple synthesis of yolk-shelled $\mathrm{ZnCo}_{2} \mathrm{O}_{4}$ microspheres towards enhancing the electrochemical performance of lithium-ion batteries in conjunction with a sodium carboxymethyl cellulose binder. J. Mater. Chem. A 2013, 1, 15292-15299. [CrossRef]

51. Bao, J.; Zhang, X.; Fan, B.; Zhang, J.; Zhou, M.; Yang, W.; Hu, X.; Wang, H.; Pan, B.; Xie, Y. Ultrathin spinel-structured nanosheets rich in oxygen deficiencies for enhanced electrocatalytic water oxidation. Angew. Chem. Int. Ed. 2015, 127, 7507-7512. [CrossRef]

52. Jia, G.; Hu, Y.; Qian, Q.; Yao, Y.; Zhang, S.; Li, Z.; Zou, Z. Formation of hierarchical structure composed of (Co/Ni)Mn-LDH nanosheets on MWCNT backbones for efficient electrocatalytic water oxidation. ACS Appl. Mater. Interfaces 2016, 8, 14527-14534. [CrossRef] [PubMed]

53. Zhang, C.; Zhao, J.; Zhou, L.; Li, Z.; Shao, M.; Wei, M. Layer-by-layer assembly of exfoliated layered double hydroxide nanosheets for enhanced electrochemical oxidation of water. J. Mater. Chem. A 2016, 4, 11516-11523. [CrossRef]

54. Bikkarolla, S.K.; Papakonstantinou, P. $\mathrm{CuCo}_{2} \mathrm{O}_{4}$ nanoparticles on nitrogenated graphene as highly efficient oxygen evolution catalyst. J. Power Sources 2015, 281, 243-251. [CrossRef] 
55. Yu, M.Q.; Jiang, L.X.; Yang, H.G. Ultrathin nanosheets constructed $\mathrm{CoMoO}_{4}$ porous flowers with high activity for electrocatalytic oxygen evolution. Chem. Commun. 2015, 51, 14361-14364. [CrossRef] [PubMed]

56. Yang, F.; Sliozberg, K.; Sinev, I.; Antoni, H.; Bähr, A.; Ollegott, K.; Xia, W.; Masa, J.; Grünert, W.; Cuenya, B.R.; et al. Synergistic effect of cobalt and iron in layered double hydroxide catalysts for the oxygen evolution reaction. ChemSusChem 2016, 10, 156-165. [CrossRef] [PubMed]

57. Xie, J.; Zhang, J.; Li, S.; Grote, F.; Zhang, X.; Zhang, H.; Wang, R.; Lei, Y.; Pan, B.; Xie, Y. Controllable Disorder Engineering in Oxygen-Incorporated $\mathrm{MoS}_{2}$ ultrathin nanosheets for efficient hydrogen evolution. J. Am. Chem. Soc. 2013, 135, 17881-17888. [CrossRef] [PubMed]

58. Xie, J.; Qu, H.; Lei, F.; Peng, X.; Liu, W.; Gao, L.; Hao, P.; Cui, G.; Tang, B. Partially amorphous nickel-iron layered double hydroxide nanosheet arrays for robust bifunctional electrocatalysis. J. Mater. Chem. A 2018, 6, 16121-16129. [CrossRef]

59. Xie, J.; Li, S.; Zhang, X.; Zhang, J.; Wang, R.; Zhang, H.; Pan, B.; Xie, Y. Atomically-thin molybdenum nitride nanosheets with exposed active surface sites for efficient hydrogen evolution. Chem. Sci. 2014, 5, 4615-4620. [CrossRef]

(C) 2018 by the authors. Licensee MDPI, Basel, Switzerland. This article is an open access article distributed under the terms and conditions of the Creative Commons Attribution (CC BY) license (http:/ / creativecommons.org/licenses/by/4.0/). 